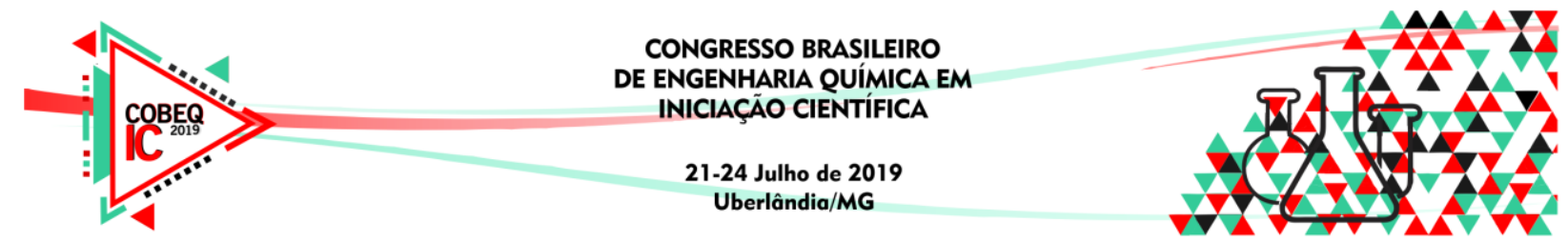

\title{
OBTENÇÃO, CARACTERIZAÇÃO E FUNCIONALIZAÇÃO DE NANOLÂMINAS DE GRAFENO PARA REMOÇÃ̃O DE TURBIDEZ DE EFLUENTE TÊXTIL
}

\author{
A. S. XAVIER ${ }^{1}$ e S. L. NOGUEIRA ${ }^{2}$ \\ ${ }^{1}$ Universidade Federal de Uberlândia, Faculdade de Engenharia Química \\ ${ }^{2}$ Centro Universitário de Patos de Minas, Curso de Engenharia Química \\ E-mail para contato: sandraln@unipam.edu.br
}

\begin{abstract}
RESUMO - A nanotecnologia é uma área que vem se destacando por suas grandes contribuições para solucionar os problemas do milênio. Um dos principais problemas que se tem atualmente é o descarte indevido de efluentes. A indústria têxtil é responsável por grande volume de efluentes líquidos com presença de contaminantes. O presente estudo propõe uma forma alternativa de tratamento de efluente têxtil utilizando nanolâminas de grafeno (NG) funcionalizadas com grupos carboxílicos para remoção de turbidez. Os experimentos foram realizados em batelada. As nanolâminas foram testadas antes e após funcionalização com diferentes massas pHs. Obteve-se até 99,17\% de redução da turbidez com as NG funcionalizadas. Além de apresentarem alto rendimento e baixo custo de produção, as NG funcionalizadas foram eficazes na remoção de turbidez.
\end{abstract}

\section{INTRODUÇÃO}

Os efeitos tóxicos da descarga de efluentes industriais em mananciais hídricos devem ser intensamente investigados. Uma vez que podem afetar a sobrevivência, o comportamento ou a composição genética dos organismos aquáticos, bem como a saúde da população, que busca nestes mananciais seu suprimento de água potável. Dentro desse contexto, a indústria têxtil gera efluentes com composição extremamente heterogênea e uma grande quantidade de material tóxico e recalcitrante, o que torna seu tratamento mais difícil (Lemos et al., 1992).

Para lidar com efluentes de composição e características variadas, meios alternativos de tratamento são constantemente investigados. Para alcançar a qualidade dentro dos parâmetros, são desenvolvidos novas técnicas e novos materiais para essa finalidade. Estudos recentes na engenharia de dispositivos com dimensões cada vez menores, chamados nanomateriais, revelam a viabilidade de seu uso no tratamento de efluentes. Dentre essas estruturas, destacam-se o grafeno, que possui uma gama de aplicações que vão da eletrônica até a área de tratamento de efluentes (Geim; Novoselov, 2007).

A funcionalização é um processo químico que consiste na inserção de grupos funcionais nas suas paredes ou extremidades com a finalidade de aumentar a compatibilidade entre materiais. A inserção de funcionalidades carboxílicas na superfície desses materiais também é uma área que vem ganhando destaque e até pouco tempo era praticamente inexplorada. As 


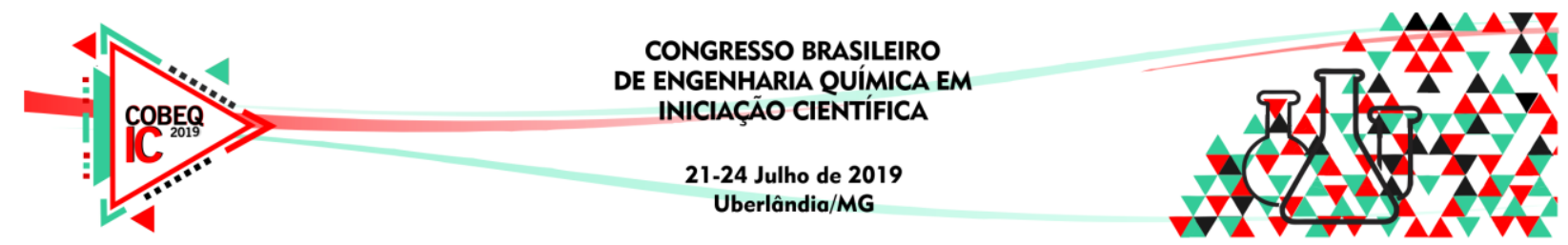

principais potencialidades da modificação com grupos carboxílicos é influenciar a reatividade e as propriedades de adsorção do material (Yang et al., 2008).

O objetivo desse trabalho consiste no estudo da produção das nanolâminas de grafeno pelo método da esfoliação química e a modificação da sua estrutura por funcionalização com grupos carboxílicos alterando suas propriedades de adsorção. Após o processo deseja-se empregar sua nova estrutura no tratamento de efluente advindo de indústria têxtil e testar sua eficácia na redução da turbidez do efluente bruto, propondo uma alternativa de aumentar a eficácia do tratamento de efluentes têxteis baseado num método viavelmente econômico.

\section{METODOLOGIA}

\subsection{Produção de NG}

A esfoliação química do grafite para produção de nanolâminas de grafeno ocorreu nas seguintes etapas: partiu-se de uma mistura 4:1 contendo ácido sulfúrico (PA), ácido nítrico (PA) e $20 \mathrm{~g}$ de grafite comercial. A mistura foi submetida a uma agitação mecânica seguida de repouso até a decantação. Nessa primeira etapa obteve-se o grafite intercalado (GI). Em seguida, retirou-se o excesso de sobrenadante e realizou-se a diluição da solução de GI decantada seguida de filtração a vácuo. $\mathrm{O}$ grafite intercalado foi aquecido a $1000^{\circ} \mathrm{C}$ durante 30s. Após esse tratamento térmico obteve-se o grafite expandido (GE). Com o GE obtido, fezse uma suspensão de álcool etílico $70 \%$ submersa em um banho de ultrassom durante $8 \mathrm{~h}$. Em seguida filtrou-se a solução e repetiu-se o processo de secagem, resultando em (NG) (Adaptado de Silva e Scarpin (2013)).

\subsection{Funcionalização de NG}

Aproximadamente $5 \mathrm{~g}$ da amostra de $\mathrm{NG}$ foi adicionada a um balão de fundo redondo de $500 \mathrm{~mL}$ contendo $400 \mathrm{~mL}$ de solução $30 \%$ v/v de peróxido de hidrogênio. O balão foi acoplado a um condensador de bolas com água circulante e, com o auxílio de uma manta aquecedora. A mistura reagente permaneceu em refluxo por 2 horas. Transcorrido este período, a mistura foi filtrada, sob vácuo, em membrana de policarbonato com tamanho de poro de $1,2 \mu \mathrm{m}$ e lavada com água destilada quente. O resíduo foi seco em estufa à vácuo, à temperatura de $120^{\circ} \mathrm{C}$, por 12 horas. O produto dessa etapa fica ligado ao grupo $\mathrm{OH}$. Após caracterização, este material foi submetido à digestão com ácido nítrico.

Em um balão de fundo redondo ( $1 \mathrm{~L})$ adicionou-se $2 \mathrm{~g}$ da amostra obtida na etapa anterior e $500 \mathrm{~mL}$ de uma solução $3 \mathrm{~N}$ de ácido nítrico. $\mathrm{O}$ balão foi vedado e submetido à sonificação em banho por 15 horas. Após este período, a mistura foi resfriada à temperatura ambiente, diluída com água destilada e filtrada sob vácuo, em membrana de policarbonato de $0,45 \mu \mathrm{m}$ de poro. Lavou-se exaustivamente com água destilada, com solução diluída de $\mathrm{NaOH}$ e novamente com água destilada até $p \mathrm{H}$ neutro. $\mathrm{O}$ resíduo foi seco em estufa à vácuo, à temperatura de $120^{\circ} \mathrm{C}$, por 12 horas (adaptado de Hu et al., 2003). Após esse processo obtevese nanolâminas de grafeno carboxiladas (NG-COOH).

\subsection{Coleta do efluente e processo batelada}




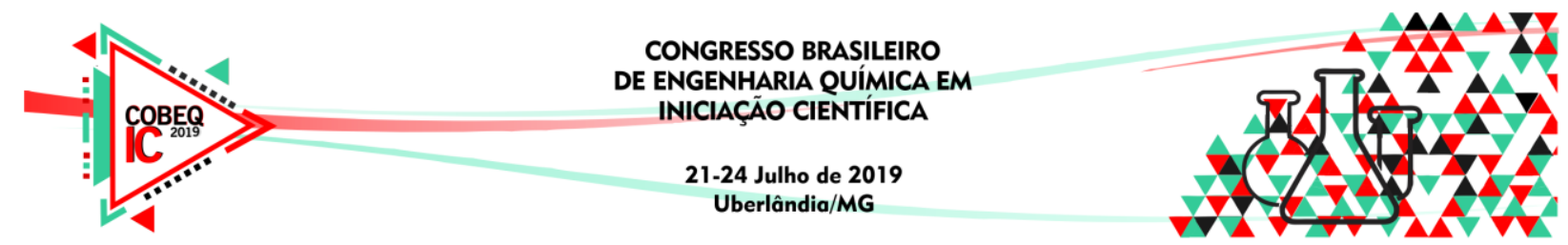

O efluente foi coletado em uma indústria têxtil localizada em Patos de Minas - MG. Para seu armazenamento utilizou-se galões de cor escura, evitando o contato com luz e umidade (para que não haja degradação dos compostos presentes). Reservou-se uma amostra desse efluente para determinação das concentrações de mercúrio, análise de turbidez e medida de $p \mathrm{H}$. Para o tratamento, avaliou-se a influência do adsorvente antes (NG) e após a funcionalização (NG-COOH), do $p \mathrm{H}$ em que se encontrava o efluente, (3, 7 e 11) como também da quantidade de massa dos adsorventes usados $(1 \mathrm{~g}$ e $200 \mathrm{mg})$. Para ajuste do $p \mathrm{H}$ do efluente, utilizou-se ácido clorídrico $(1 \mathrm{~mol} / \mathrm{L})$ e hidróxido de sódio $(1 \mathrm{~mol} / \mathrm{l})$. O experimento foi realizado em batelada e a mistura foi agitada a $50 \mathrm{rpm}$ em uma mesa de agitação orbital Vortex Mixer (modelo K45-2810). Fixou-se um tempo de agitação de $1 \mathrm{~h}$ e $100 \mathrm{~mL}$ de efluente para todos os testes. Após a agitação, a mistura foi submetida à filtração simples e retirou-se uma alíquota de cada amostra para análises de turbidez.

\section{RESULTADOS E DISCUSSÕES}

\subsection{Raman}

A Figura 1 apresenta os espectros Raman para GR (grafite), GI (grafite intercalado), GE (grafite expandido) e NG (nanolâminas de grafeno). Todos os espectros exibem picos característicos na região entre 1350 e $3000 \mathrm{~cm}^{-1}$ para energia de excitação do laser de argônio em $514 \mathrm{~nm}(2,41 \mathrm{eV})$. As bandas encontradas neste intervalo são conhecidas como banda D (1200 a $\left.1400 \mathrm{~cm}^{-1}\right), \mathrm{G}\left(1500\right.$ a $\left.1650 \mathrm{~cm}^{-1}\right)$ e $2 \mathrm{D}\left(2600 \mathrm{a} 2750 \mathrm{~cm}^{-1}\right)$.

Figura 1 - Espectro Raman para as amostras GR, GI, GE e NG.

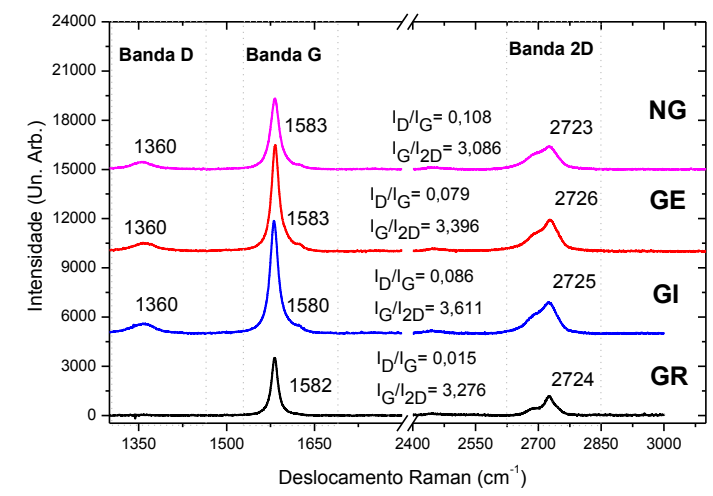

A banda $\mathrm{G}$ está relacionada as vibrações $\mathrm{C}-\mathrm{C}$ do grafite, as quais estão presentes nos materiais de carbono. A banda $\mathrm{D}$ tem relação com a desordem da estrutura hexagonal do grafite. Como a banda D está associada à pobre grafitização, ela é conhecida como a banda da "desordem" ou banda amorfa. Conforme apresentado na Figura 1, o sinal da banda D é menos intenso para o grafite testado. Para as demais amostras o sinal da banda D alcança a intensidade de $1360 \mathrm{Un}$. Arb., indicando materiais estruturalmente mais desordenados. Isso se deve ao processo de esfoliação química do grafite que introduz defeitos em sua estrutura cristalina em razão da quebra grafítica como também a incorporação de grupos oxigenados pela oxidação do grafite no tratamento com ácido forte.

Uma maneira de quantificar esta desordem é a partir da razão das intensidades das bandas $\mathrm{D}$ e $\mathrm{G}$ (valor $\mathrm{I}_{\mathrm{D}} / \mathrm{I}_{\mathrm{G}}$ ). De acordo com a literatura, (Ferrari et al, 2012), o valor de $\mathrm{I}_{\mathrm{D}} / \mathrm{I}_{\mathrm{G}}$ próximo de zero é um indicativo de material grafítico cristalino, quando essa razão aumenta, o 


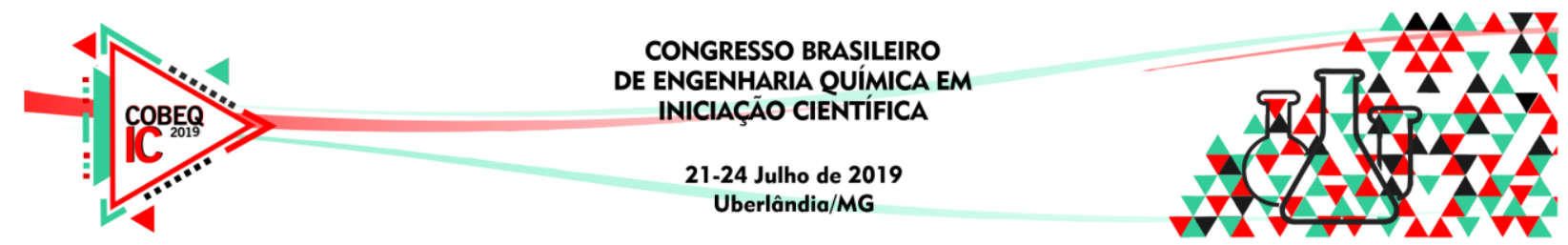

material grafítico perde sua cristalinidade e torna-se estruturalmente mais desorganizado. A razão de intensidade das bandas é mostrada na Figura 1. O grafite empregado neste estudo apresentou elevado grau de cristalinidade $(\mathrm{ID} / \mathrm{IG}=0,015)$. Entretanto, os valores de $\mathrm{I}_{\mathrm{D}} / \mathrm{I}_{\mathrm{G}}$ bem próximos para GI, GE e NG revelam a presença de maior quantidade de defeitos estruturais. A partir da banda 2D torna-se possível a identificação de nanolâminas de grafeno no material, e sobretudo, a quantidade de nanolâminas (Ferrari et al, 2006). O número de camadas pode ser obtido a partir da razão das intensidades da banda $2 \mathrm{D}$ e da banda $\mathrm{G}$ (valor $\mathrm{I}_{\mathrm{G}} / \mathrm{I}_{2 \mathrm{D}}$ ). Na Figura 1 nota-se que o espectro que apresentou menor valor para a razão $\mathrm{I}_{\mathrm{G}} / \mathrm{I}_{2 \mathrm{D}}$ foi a amostra de NG. Os espectros das amostras analisadas apresentam características estruturais diferentes de um material grafítico. É possível concluir que houve a formação de nanolâminas a partir da esfoliação de grafite.

\subsection{Infravermelho}

Para caracterizar a eficiência da funcionalização com os grupos carboxílicos, as NG$\mathrm{COOH}$ foram submetidas à análise de espectroscopia na região do infravermelho. Esta é uma ferramenta poderosa na caracterização dos grupos funcionais adicionados em materiais nanoestruturados (Nogueira et al., 2012). A Figura 2 mostra os resultados de infravermelho para NG e NG-COOH.

Figura 2 - Infravermelho para as amostras de NG e NG-COOH.

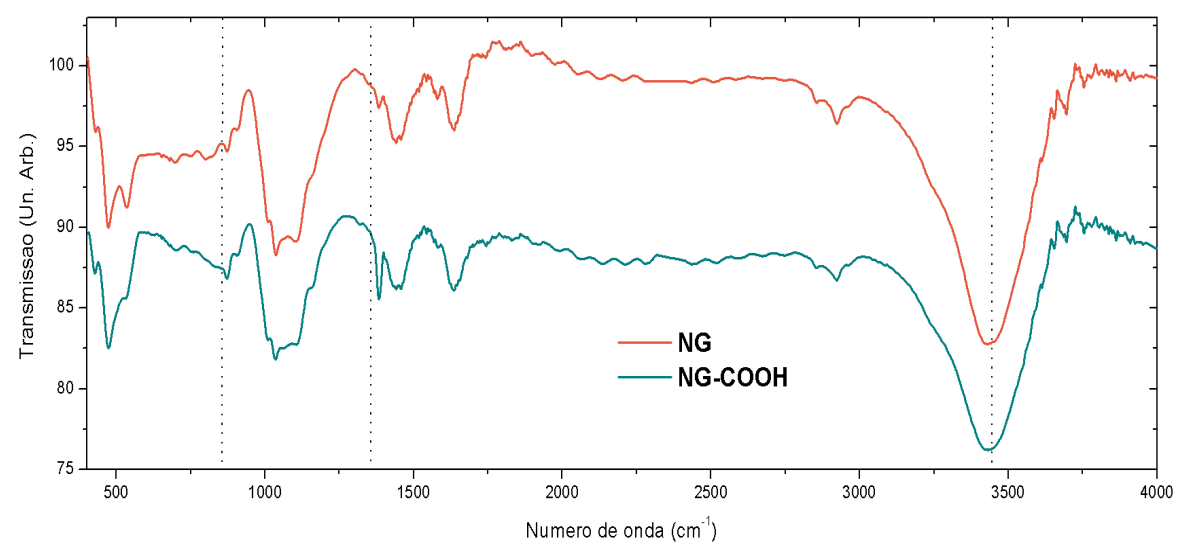

De acordo com a literatura, a presença de bandas de absorção compreendidas entre 600 e $1000 \mathrm{~cm}^{-1}$ indicam a presença da ligação $\mathrm{C}=\mathrm{C}$ nas estruturas (Silverstein, 2007). De acordo com a Figura 2, ambas as amostras apresentaram esta banda., indicando a formação eficaz da estrutura de carbono. Por volta de $500 \mathrm{~cm}^{-1}$ observa-se a presença de um pico para amostra NG. Este pico não é observado para NG-COOH, o que pode ser atribuída a quebra dos anéis de carbono durante o processo de funcionalização. Bandas de absorção observadas entre 1400 e $1600 \mathrm{~cm}^{-1}$ no espectro, evidenciam a presença de grupos carbonílicos $(\mathrm{C}=\mathrm{O})$ (Silverstein, 2007). Para NG-COOH nota-se um pico mais intenso nesse intervalo que não é observada em NG, confirmando a inserção dos mesmos na estrutura após funcionalização. Já a presença de grupos O-H ligados à estrutura é evidenciada pelo aparecimento de bandas de absorção alargadas próximas à $3400 \mathrm{~cm}^{-1}$ (Silverstein, 2007). Em NG a identificação dessa banda indica a presença de hidroxilas advindos de esfoliação química e em NG-COOH reafirma a inserção dos grupos carboxílicos. 


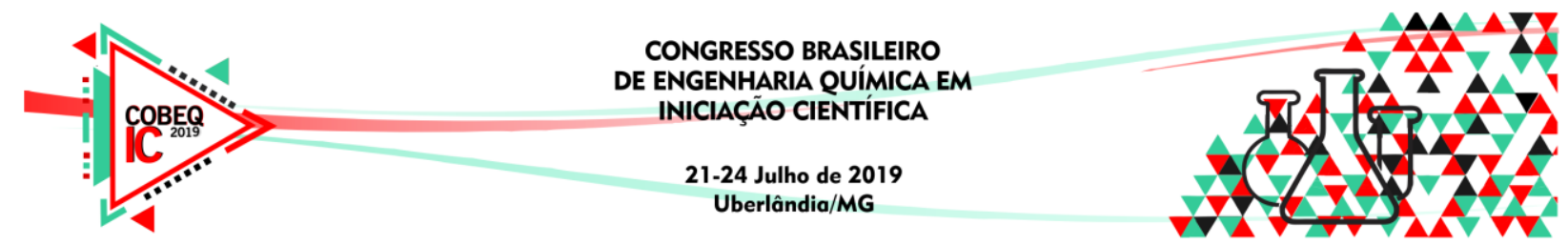

\subsection{Remoção de turbidez}

A turbidez das amostras foi lida em um turbidímetro portátil, no laboratório de Engenharia Química do Centro Universitário de Patos de Minas. Foram comparados os valores de turbidez do efluente bruto e do efluente após tratamento. A porcentagem de remoção de turbidez para cada condição testada é apresentada na Tabela 1. A descrição das amostras é constituída pelo adsorvente utilizado (NG-antes da funcionalização e NG-COOHapós a funcionalização), pelo $p \mathrm{H}$ de trabalho e pela massa utilizada de cada adsorvente, respectivamente.

Tabela 1 - Remoção da turbidez.

\begin{tabular}{|c|c|}
\hline Descrição das amostras & Remoção da turbidez [\%] \\
\hline NG- $p \mathrm{H} \mathrm{3-1} \mathrm{g}$ & 97,11 \\
\hline NG- $p \mathrm{H} 7-1 \mathrm{~g}$ & 98,09 \\
\hline NG- $p \mathrm{H} \mathrm{11-1} \mathrm{g}$ & 97,45 \\
\hline NG- $p \mathrm{H}$ 3-200 mg & 98,27 \\
\hline NG- $p \mathrm{H}$ 7-200 mg & 98,03 \\
\hline NG- $p \mathrm{H} 11-200 \mathrm{mg}$ & 97,62 \\
\hline NG-COOH- $p \mathrm{H} 3-1 \mathrm{~g}$ & 99,07 \\
\hline NG-COOH- $p \mathrm{H}$ 7-1 g & 99,24 \\
\hline NG-COOH- $p \mathrm{H} 11-1 \mathrm{~g}$ & 98,54 \\
\hline NG-COOH- $p \mathrm{H} 3-200 \mathrm{mg}$ & 99,09 \\
\hline NG-COOH- $p \mathrm{H}$ 7-200 mg & 99,17 \\
\hline NG-COOH- $p \mathrm{H} 11-200 \mathrm{mg}$ & 98,55 \\
\hline
\end{tabular}

De acordo com a Tabela 1, as combinações com o nanomaterial funcionalizado resultaram na maior remoção de turbidez comparado ao nanomaterial sem grupo carboxílico. Com isso, pode-se dizer que a carboxilação aumentou a propriedade de remoção da turbidez, tornando viável sua utilização pra esse fim. Essa capacidade de remoção das NG-COOH foi explicado por Wang et al., (2012), que constatou que grafeno carboxilado se liga aos sólidos suspensos presentes e decanta juntamente com eles, facilitando sua filtração.

Maiores porcentagens de remoção foram obtidas com o adsorvente em meios ácidos e neutros (Tabela 1). Esse fato é influenciado pela rota de síntese das NG e sua funcionalização com COOH. Em ambos os casos, ácidos são utilizados para intercalação e reação de ligação com os grupos carboxílicos. Desse modo, a estrutura dos adsorventes é perturbada quando em meio alcalino e prejudica sua propriedade de se ligar às impurezas (Kuila et al., 2012).

\section{CONCLUSÕES}

Através da espectroscopia Raman foi possível identificar bandas características de nanolâminas de grafeno, constatando sua formação eficaz. Pelo espectro infravermelho identificou-se a presença dos grupos inseridos na funcionalização (carboxílicos) afirmando a funcionalização das NG. 


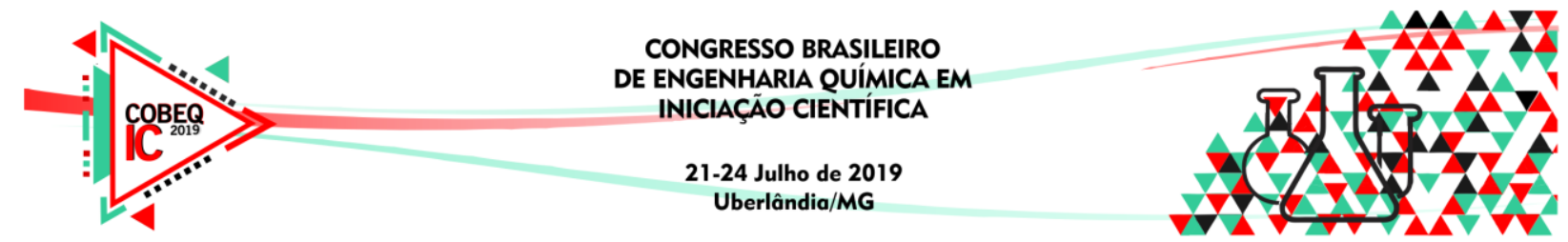

Além disso, as NG apresentaram grande potencial de remoção de turbidez, principalmente quando funcionalizadas. Nesse sentido, comprovou-se a viabilidade das funcionalizações para esse tipo de tratamento e seu uso em indústrias têxteis Nesse sentido, comprovou-se a viabilidade das funcionalizações para esse tipo de tratamento e seu uso em indústrias têxteis.

\section{REFERENCIAS}

FERRARI, A. C. et al. Síntese e Propriedades de Nanocompósitos de Polietileno/Nanolâminas de Grafeno Obtidos Através de Polimerização In Situ. 2012. 90p. Tese (Doutorado) - Faculdade de Ciência dos Materiais, Universidade Federal do Rio Grande do Sul, Porto Alegre, 2012.

FERRARI, A. C. et al. Raman Spectrum of Graphene and Graphene Layers. PRL, 2006.

GEIM, A. K.; NOVOSELOV, K. S. The rise of graphene. Nature Materials, v. 6, p. 183-191, 2007.

HU, H. et al. Nitric acid purification of single-walled carbon nanotubes. Journal of Physical and Chemistry, v.107, n.50, p.13838 - 13842, 2003.

KUILA, T. et al. Chemical functionalization of graphene and its applications. Progress in Materials Science, v. 57, p. 1061-1105, 2012.

LEMOS, A.M. et al. Comparative Performance of six Holstein-Friesian x Guzera grades in Brazil.5. Age atfirstcalving. Revista Brasileira de Genética, v.15, n.1, p.73-83, 1992.

NOGUEIRA, S. L.; ARMOND, R. A. da S. Z.; FURTADO, C. A.. Síntese e caracterização de Sistemas Orgânicos Semicondutores Baseados em Tiofeno-Fenileno para Aplicação em Células Fotovoltaicas. 2012. 231 f. Tese (Doutorado) - Curso de Física, Universidade Ferderal de Uberlândia, Uberlândia, 2012.

SILVA, M. F.; PINEDA, E. A. G.; BERGAMASCO, R. OLIVEIRA, C. A. Aplicação de óxidos de ferro nanoestruturados como adsorventes e fotocatalisadores na remoção de poluentes de águas residuais. Química Nova, v. 38, n. 3, p. 393-398, 2014.

SILVERSTEIN, R. M.; Bassler, G. C.; Morrill, T. C. Identificação espectrométrica de compostos orgânicos. 3 ed. Rio de Janeiro: Editora Guanabara Dois S.A., p. 1-10, 6573, 1979.

WANG, H., et al. A novel biosensing strategy for screening G-quadruplex ligands based on graphene oxide sheets. Biosensorsand Bioelectronics, v. 34, p. 88-93, 2012

YANG, F. O. et al. Chemical Functionalization of Graphene Nanoribbons by Carboxyl Groups on Stone-Wales Defects. J. Phys. Chem. C, v.112, p. 12003-12007, 2008. 